\title{
Vehicle Starter using Face Recognition
}

\author{
B. V. Subbayamma, U. Durga Srihitha, S. Mohini Sekunthala
}

\begin{abstract}
Face acknowledgment is an interesting exploration subject as of late. The scientists proposed different strategies. The factors are similar to an assortment of lighting up, outward appearance, leveling, and perspective turn of events influences the precision of the face affirmation procedure. The fundamental requirement is the separation of the facial picture and the SURF (Speeded up Robust Features). Notwithstanding that they are additionally halfway invariable to brightening and relative change. This undertaking recommends a facial acknowledgment procedure utilizing SURF highlights and Support Vector Machine (SVM) classifier. The outcomes demonstrate that the proposed technique can prompt high acknowledgment productivity. The proposed framework is applied to vehicle get to control by interfacing the Arduino microcontroller board with PC.

Index terms: SURF, SVM, SIFT, RBF
\end{abstract}

\section{INTRODUCTION}

Presently nowadays there is a remarkable enthusiasm for sound and video observation. Along these lines, it is continually an inciting task to think about a capable and consistent figuring for faster recognizing, following, and affirmation of the face. If we can achieve the endeavor of doing snappier the route toward recognizing, following, and affirmation of the face, by then it might be used in various applications like security structure where obliged people can be allowed to go to certain districts whereas others are not constrained or confined. The apparatuses that we used for the method are MATLAB and Arduino. The clarification behind using MATLAB is a direct result of its wide extent of innate limits in Image Processing and it is in like manner easy to interface it with Arduino. The utilization of Arduino is its ability to control the advancement of Motors. The correspondence way used while interfacing MATLAB with Arduino is the Serial kind of Communication. Various computations that are used to perceive and follow a face. At present computation used for distinguishing and following on the face is Viola_Jones estimation. The methodology used for face affirmation is SURF Features and SVM grouping. Face acknowledgment has been explored from the last many decades. Face affirmation targets seeing a given picture from an image databank. Consequently, we need to initially decide the facial zone.

Revised Manuscript Received on June 08, 2020.

* Correspondence Author

B. V. Subbayamma*, Assistant Professor, Department of ECE, Prasad V. Potluri Siddhartha Institute of Technology, Kanuru, Vijayawada, Andhra Pradesh, India.

U. Durga Srihitha, Department of ECE, Prasad V. Potluri Siddhartha Institute of Technology, Kanuru, Vijayawada, Andhra Pradesh, India.

S. Mohini Sekunthala, Department of ECE, Prasad V. Potluri Siddhartha Institute of Technology, Kanuru, Vijayawada, Andhra Pradesh, India.

(C) The Authors. Published by Blue Eyes Intelligence Engineering and Sciences Publication (BEIESP). This is an open access article under the CC BY-NC-ND license (http://creativecommons.org/licenses/by-nc$\underline{\mathrm{nd} / 4.0 /)}$
At that point, some all-around characterized features are recognized (discovery of feature) from the picture. These features ought to be Volume-9 Issue-5, June 2020 addressed as courses with mathematical qualities to coordinate with an incorporated course of other pictures. There are numerous obstructions to achieve a great affirmation degree. The analysts introduce some nearby features to deal with these concerns. Nearby features, for instance, corners and masses are generally used for object affirmation. Generally the arena of face affirmation using SIFT (Scale Invariant Feature Transform) introduced by D. Lowe [1] [2]. In any case, meanwhile, its imagining time is great it constrains the speed in alive picture applications. SVM (Support Vector Machine) is a generally utilized strategy for information arrangement similarly as backslide. We have utilized SURF includes as highlights of the picture as SVM as a classifier. Be that as it may, rather than doing characterization of face pictures overall we have done component insightful order to choose the name of the picture. The second portion is a prologue to SVM and SURF. Third fragment portrays the proposed strategy. Fourth portion displays the outcomes and the fifth fragment is an end.

\section{IMPLEMENTATION}

The core of the Speeded up Robust Features identification is non-maximal concealment of the factors of the hessian frameworks. The complication approached and speeded-up with the utilization of indispensable pictures and it is exorbitant to compute [3]. The hessian grid must be discretized and trimmed using Gaussian kernels22()gx. The SURF calculation approaches these bits through quadrilateral boxes and box screens. Similar to SIFT, the SURF fabricates a pyramid scale space, for scale invariance. The next step is to intrigue point depiction. SURF utilizes the total of the Haar wavelet reactions to depict the element of an intriguing argument [2]. Fig. 2 displays the Haar wavelet channels to figure reactions at $\mathrm{x}$ and $\mathrm{y}$ headings. For the abstraction of the descriptor, the initial phase comprises building a square locale focused at the intriguing argument and arranged lengthwise direction using the direction choice strategy [2].

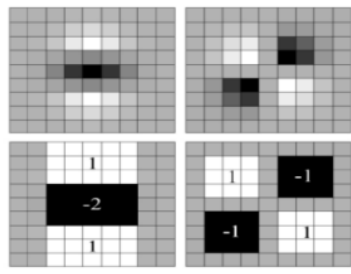

Figure 1: Discretized and trimmed Gaussian Second request halfway subordinates and their calculations [3].

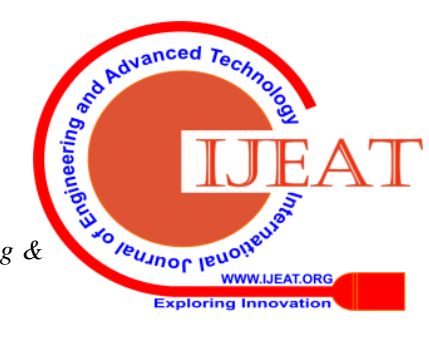




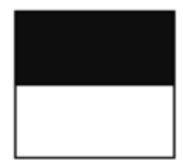

$d y$

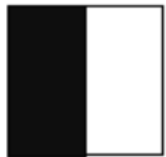

$d x$
Figure 2: Haar wavelet channels for the estimation of reactions on $x$ and $y$ orders.

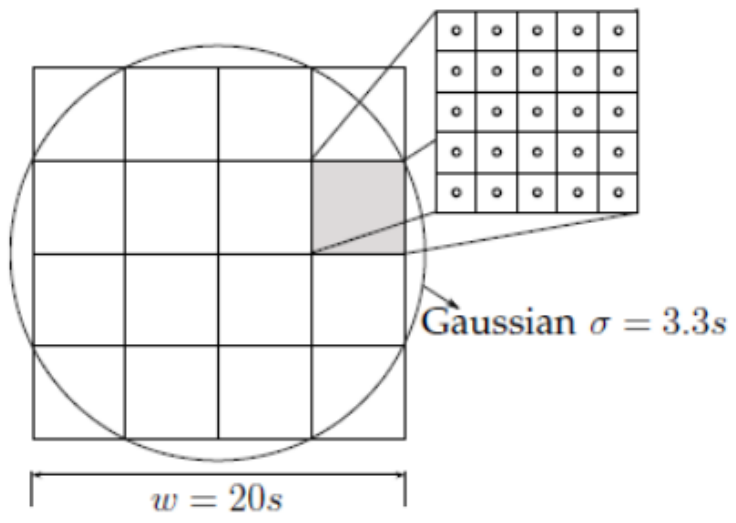

Figure 3: The wavelet reaction [3] for the 20s spaces are separated as $4 \mathrm{X} 4$ subparts that are tested $5 \times 5$ periods.

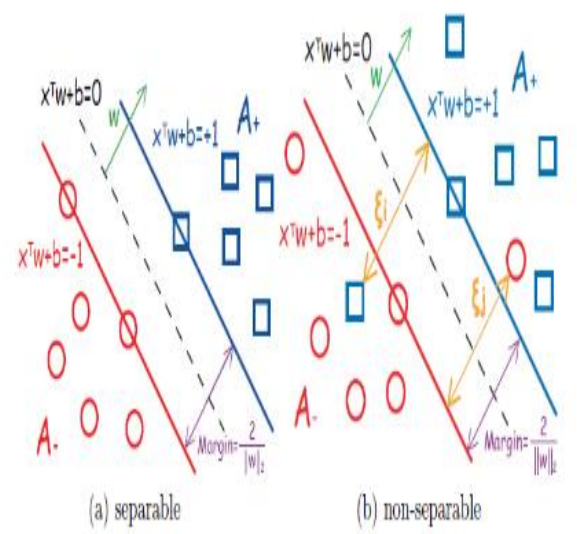

Figure 4: Straight SVM for distinct and nondistinguishable information [5].

\section{METHODOLOGY}

In this methodology Speeded up Robust Features highpoints of face, pictures are removed and coordinated utilizing SVM strategy. Right now take hardly any planning pictures to prepare the SVM sorter. A data bank is made with facial SURF highlights of preparing pictures of a considerable number of people. For another picture (individual), above all else, the facial district will be recognized (in case it's not an altered face picture). For distinguishing face locale, ViolaJones calculation is used for face discovery [6]. At that point, the highlights of the SURF are recognized for the face district. The yield of this progression is the SURF arguments article, comprising data of the SURF highlights recognized in the input picture of 2-D grayscale. After this progression include abstraction will be finished. This progression proceeds removed component segments, otherwise called signifiers. The dimension of this component segment is $\mathrm{mx} 64$, where $\mathrm{m}$ is the quantity of extricated include focuses.
Presently this element vector will be ordered utilizing SVM sorter.

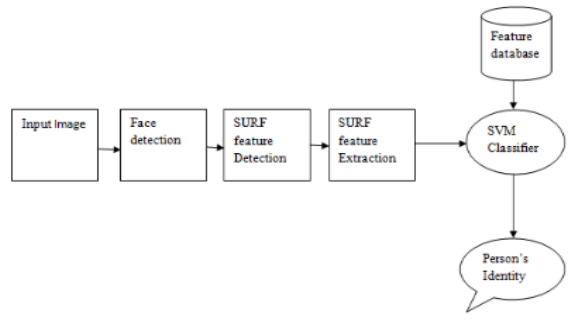

Figure 5: Proposed method for face recognition

Presently during characterization, prepared our sorter other than the separate face images all in all but rather as far as the highlights. For instance as opposed to naming a specific picture as $r$, where $r$ is a number. The portion processes the speck item which would some way or another be substantially more costly too as r. Along these lines on the off chance that we get $n$ highlights from an example, at that point n columns (include vector) will be named as $r$. Thus while testing likewise as opposed to foreseeing mark of the testing picture overall names of acquired highlights are anticipated. On the off chance that the name of a larger part of highlights is coordinating with that the expected mark then it is an effective acknowledgment. We have utilized the $\mathrm{RBF}$ part for grouping. The factor gamma and $\mathrm{C}$ of $\mathrm{RBF}$ portion streamlined by Cross-approval and Grid-Search strategy. Framework satisfies all the normal outcomes and it is productive for the user. The framework can be utilized.

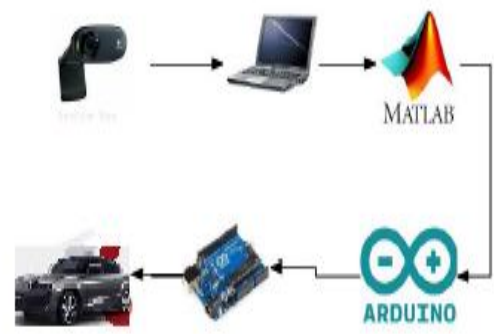

Fig 6 overall proposed system block diagram

\section{RESULTS AND DISCUSSION}

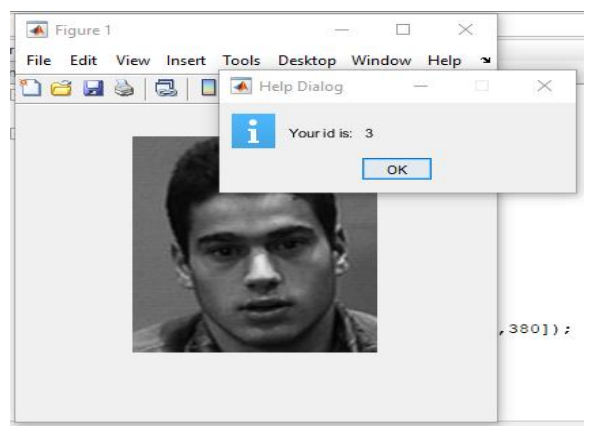

Fig 7 Authorised face

Published By:

Blue Eyes Intelligence Engineering \& 
SURF highlights give an evident favorable position of uniform moving, revolution and calibrating, and incomplete steady to illumi country and relative change. Practically the same focal points are likewise given by SIFT highlights. However, the SURF is a lot quicker than SIFT for include recognition and highlight abstraction. The Support Vector Machine (SVM) sorter is an incredible grader that functions admirably on a wide scope of arrangement issues, even issues in high measurements that are not directly distinguishable.

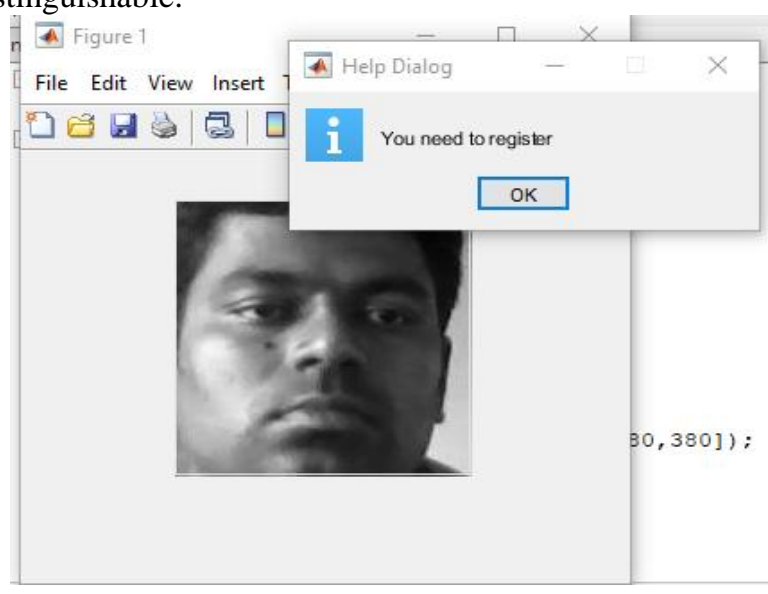

Fig 8 Unauthorised face

When we need to manage a multi-faceted information Support Vector Machine is fit in those situations. For our situation, every element is of 64-measurement. At that point, each class has numerous highlights. Subsequently by and large get a multi-faceted element dataset then SVM is a decent other option. Outcomes display a decent precision through the pictures having variety in enlightenment, viewpoint development, outward appearance, and scaling. In the future, we can also think of with picture pretreating for enhanced brightening and dilatation. At last with face affirmation module is applied to vehicle start control by interfacing with Arduino.

\section{FUTURE SCOPE}

From the outcomes got in the show it is clear the framework gives quicker face recognition and acknowledgment for proprietor authentication. Ignition is given quickly, after proprietor validation, for beginning the vehicle engine. This implies that the framework satisfies all the normal outcomes and it is productive for the user. The framework can be utilized to lessen the expanded vehicle burglary and permits the proprietor to distinguish the gatecrasher subsequently having the vehicle under his/her control. The results acquired through the face acknowledgment show that it very well may be depended upon to guarantee the wellbeing of the vehicle. The framework is additionally solid to be utilized in other approval applications including apply autonomy, outskirt the board, banking security including ATMs, and so on.

\section{CONCLUSION}

SURF structures deliver the apparent benefit of invariability in change, pivot and leveling, and fractional steady to illumi country and relative change. Practically the same favorable circumstances are likewise given by SIFT highlights. In any case, SURF is a lot quicker than SIFT regarding highlight discovery and highlight abstraction. The SVM is a dominant sorter for an extensive scope of grouping issues, even issues in high measurements and that are not directly detachable. SVM is fit in the situations when we have to manage exceptionally multi-faceted information. For our situation, each component is of 64-measurement. Henceforth in general we get a large-sized element databank. In such cases, SVM can be a decent other option. The outcomes display a decent exactness with the pictures having variety in brightening, viewpoint development, outward appearance and, scaling. In the future, we can also think of comprising image pretreating so for better illumination and dilatation. Finally with face recognition module is applied to vehicle ignition control by interfacing with Arduino.,

\section{REFERENCES}

1. D. Lowe, "Distinctive image features from scale-invariant keypoints, cascade filtering approach", International Journal of Computer Vision, vol 60, pp 91-110, January 2004.

2. Lowe, D.G. 1999. Object recognition from local scale-invariant features. In International Conference on Computer Vision, Corfu, Greece, pp. 1150-1157.

3. H. Bay, A. Ess, T. Tuytelaars, L. Van Gool, "Speeded-up robust features (SURF)", Comput. Vis. Image Underst., 110(3), 346-359 (2008).

4. A technical report on Feature detection and implementing the Speeded-Up Robust Features(SURF) algorithm.

5. Yuh-Jye Lee, Yi-Ren Yeh, and Hsing-Kuo Pao, An Introduction to Support Vector Machines, National Taiwan University of Science and Technology, Taipei, Taiwan.

6. M. Jones and P. Viola, Face Recognition Using Boosted Local Features,

7. IEEE International Conference on Computer Vision, 2003

\section{AUTHORS PROFILE}

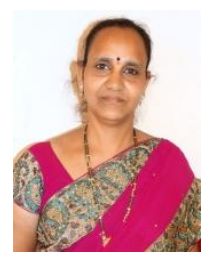

B.V.Subbayamma received UG degree from VRS Engineering College, Vijayawada, Andhra Pradesh. She received PG degree in the stream of Communications and Radar systems from VRSEC, Vijayawada, Andhra Pradesh. Currently, she is working as Assistant Professor in the Department of ECE, Prasad V. Potluri Siddhartha Institute of Technology, Kanuru, Vijayawada. Her areas of interest include Communications and Image

Processing. She is a member of IETE \& ISTE.

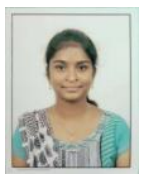

U. Durga Srihitha is pursuing Bachelor of Technology in

the Department of ECE, Prasad V.Potluri Siddhartha Institute of Technology, Kanuru, Vijayawada. She is a member of the IETE Student Forum. She is also CLAD certified.

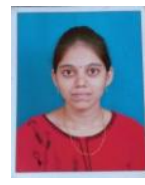

S.Mohini Sekunthala is pursuing Bachelor of

Technology in the Department of ECE, Prasad V.Potluri

Siddhartha Institute of Technology, Kanuru, ijayawada.

also

She is a member of the IETE Student Forum. She is

CLAD certified.

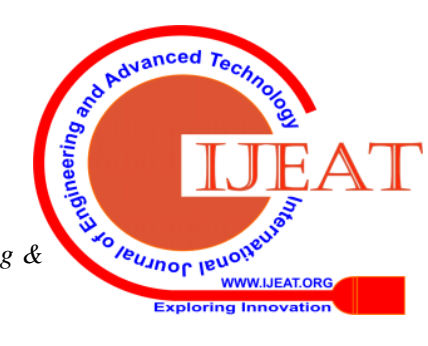

